\title{
A Robust Evolutionary Algorithm for Optical Thin-Film Designs
}

\author{
Jinn-Moon Yang \\ Department of Computer Science \\ and Information Engineering, \\ National Taiwan University, \\ Taipei, Taiwan \\ moon@csie.ntu.edu.tw
}

\author{
Cheng-Yen Kao \\ Department of Computer Science \\ and Information Engineering, \\ National Taiwan University, \\ Taipei, Taiwan \\ cykao@csie.ntu.edu.tw
}

\begin{abstract}
-
This paper presents an evolutionary approach, called Family Competition Evolutionary Algorithm (FCEA), for optical thin-film designs. The proposed approach, based on family competition and multiple adaptive rules, integrates decreasing-based Gaussian mutation and two selfadaptive mutations to balance the exploitation and exploration. It is implemented and applied to two coating systems. Numerical results indicate that the proposed approach is very robust for optical coatings.
\end{abstract}

\section{Introduction}

The optical thin-film coating is technologically very important to modem optics. It can be broadly described as follows [20], [10]: any device or material is deliberately used to change the spectral intensity distribution or the state of polarization of the electromagnetic radiation incident on it in order to satisfy performance specification and some constraints. Optical thin-film coatings have numerous remarkable applications in many branches of science and technology, such as scientific instrument manufacturing, spectroscope, medicine, and astronomy. They also have used in architecture, the automotive industry, energy conversion, computer and display devices, and communication [11].

Many approaches have been proposed to design optical thin-film coating systems. They can be divided roughly into three categories [10], [20]: analytical, graphical, and numerical methods. The numerical methods are particularly powerful because they can be applied to the design of coatings with much more complicated properties than the other methods. The problem of an optical coating design can be formulated as an optimization problem based on the use of merit functions [9]. Generally, this optimization problem is extremely difficult because of the large number of local minimum in the merit function.

Refinement methods [1], [12] and synthesis methods [8], [19], [26] are two widely used numerical methods for optical thin-film designs. Refinement methods, such as damped least square, modified gradient, golden section, Hook and Jeeves search, and simplex method, normally require a starting design that is closed to the desired performance [1], [12]. And then a desired solution is achieved by gradually modifying from the staring design. The solution quality of refinement methods heavily depends on and is sensitive to the start point. Unfortunately, good starting designs are not readily available for many modern design problems. At the same time, to choose a good starting design is time-consuming and difficult tasks in a complexity coating system.

Contrary to refinement methods, synthesis methods [19] such as gradient evolution, flip-flop method, inverse Fourier transform [7], and simulated annealing [27] generate their own starting designs automatically. They are usually combine with numerical refinement methods due to their solution quality is often not enough well. Some of synthesis and refinement methods require the determination of first and second derivation of the merit function. Therefore, to develop a good synthesis method is an important research topic.

More recently, evolutionary algorithms [15], [22], [18], [29] have been applied to optical thin-film designs. Evolutionary algorithms [17], [4], [16] represent an emerging class of algorithms which are able to provide optimal or nearoptimal solutions to a wide range of challenging problems. Their basic ideas are borrowed from genetics and natural selection. They are a generally adaptable concept for problem solving, especially well suited for solving difficult optimization problems where traditional optimization methods are less efficient. Evolutionary algorithms have been successfully applied to many problems encountered in optical filters and coatings which are inherently computational complexity. These articles demonstrated that evolutionary algorithms are robust and obtain competitive results with others synthesis methods. There are about three main independently developed but strongly related implementations of evolutionary algorithms: genetic algorithms [17], evolution strategies [4], and evolutionary programming [16].

A bit-string genetic algorithm [15] used a simple and fixed five-layer structure to design a silver-based heat system. An evolution strategy [18] was applied to design a filter used in colorimetry. In [21], [22] authors combined a gradient method into a real-coded genetic algorithm for synthesizing three complex inhomogenous problems. Their results are very competitive with the ones obtained by non-evolutionary approaches. A mixed-integer evolution strategies technique used self-adaptive mutations to solve the homogeneous coating problems [5], [23]. All of these evolutionary algorithms optimize a synthesis system with a fixed number of layers.

In this paper, we used called family competition evo- 
lutionary algorithm (FCEA) [31], [30] to synthesize multilayer thin-film systems with various number of layers. FCEA combines decreasing-based Gaussian mutation, self-adaptive Gaussian mutation, and self-adaptive Cauchy mutation. It incorporates family competition and adaptive rules to construct the relationship between mutations whose performance heavily depends on the same factor, called step size. FCEA integrates self-adaptive mutations, adapting the step sizes with a stochastic mechanism, with decreasing-based mutations, decreasing the step sizes with fixed rate $\gamma$ where $\gamma<1$, by using the family competition and adaptive rules to balance the exploration and exploitation. The family competition is derived from $(1+\lambda)$-ES [4] and acts as a local search procedure.

The rest of this paper is organized as follows. Section 2 describes the optical thin-film coating problems. Section 3 introduces the evolutionary nature of FCEA. In Section 4 two coating designs are presented to illustrate performance of our proposed approach. We also compare FCEA with various approaches on these problems. Concluding comments are drawn in Section 5.

\section{Problem Definition}

The problem in numerical design of optical multilayer coatings is to find the construction parameters of systems which satisfy the desired optical specification. The construction parameters includes the number of layers $(M)$, the thickness $(d)$, refractive indices $(\eta)$, and extinction coefficients $(k)$ of the medium, substrate, and layers. In general, synthesis methods are to find thickness $\left(d_{1}, \cdots, d_{M}\right)$ and indices $\left(\eta_{l}, \cdots, \eta_{M}\right)$ of $M$ layers. Fig. 1(a) shows a thin-film system consisting of $M$ layers.

Before you design a multilayer coating system, it is necessary to define the requirements. Most often this is defined by specifying the target transmittance $\hat{T}$ or target reflectance $\hat{R}$ at a number of wavelengths in the interesting spectral region. If the materials in a multilayer coating system are all nonabsorbing, then $\hat{T}=1-\hat{R}$. The purpose of designing a thinfilm system is to find the number of layers $M$, the refractive indices $\eta_{j}$, and the thickness $d_{j}$ of the $j$ th layer, in order to match closely the specified performance where $1 \leq j \leq M$. A coating system is called normal-incidence coating if the incident angle $\theta_{0}$ shown in Fig. 1(a) is zero, otherwise it is called oblique-incidence coating.

Let the spectral reflectance of the $M$-layer system shown in Fig. 1(a) be denoted as $R(\eta, d, \lambda)$ where $\lambda$ is the interesting wavelength region. The desired spectral reflectance profiles are fitted by minimizing a suitable merit function [20] which composes of an appropriate function of $R(\eta, d, \lambda)$ defined within the wavelength range of interesting region: $\left[\lambda_{l}, \lambda_{u}\right]$ where $\lambda_{l}$ and $\lambda_{u}$ are low and upper bound wavelength of interesting spectrum region. A widely working merit function can be defined in the following equation:

$$
f(\eta, d, \lambda)=\int_{\lambda_{l}}^{\lambda_{u}}\left\{[R(\eta, d, \lambda)-\hat{R}(\lambda)]^{2}\right\}^{1 / 2} d \lambda
$$

where $\hat{R}(\lambda)$ is the target reflectance. Fig. 1(b) shows an example of a target reflection $(\hat{R}(\lambda))$ and a respective design reflection $(R(\eta, d, \lambda))$. In computer practice, this integral is approximated by a summation over a discrete number $W$ of wavelength value $\lambda_{k}$ and can be represented as

$$
f\left(\eta, d, \lambda_{k}\right)=\Sigma_{k=1}^{W}\left\{\left[R\left(\eta, d, \lambda_{k}\right)-\hat{R}\left(\lambda_{k}\right)\right]^{2}\right\}^{1 / 2} .
$$

The most general method of calculating $R\left(\eta, d, \lambda_{k}\right)$ is based on a matrix formulation which is useful especially when the number of optimizing parameters is large. According to the matrix method, the reflectance $R\left(\eta, d, \lambda_{k}\right)$ of a multilayer system at wavelength $\lambda_{k}$ is given by

$$
R\left(\eta ; d, \lambda_{k}\right)=\left|\frac{\eta_{a} E_{k}-H_{k}}{\eta_{a} E_{k}+H_{k}}\right|^{2}
$$

where $\eta_{a}$ is refractive index of incident medium. $E_{k}$ and $H_{k}$, the electric and magnetic vector, respectively, are defined as

$$
\left[\begin{array}{l}
E_{k} \\
H_{k}
\end{array}\right]=\left(\prod_{j=1}^{M}\left[\begin{array}{cc}
\cos \theta_{j} & i \eta_{j}^{-1} \sin \theta_{j} \\
i \eta_{j} \sin \theta_{j} & \cos \theta_{j}
\end{array}\right]\right)\left[\begin{array}{c}
1 \\
\eta_{s}
\end{array}\right]
$$

where $\theta_{j}=\frac{2 \pi}{\lambda_{k}}\left(n_{j} d_{j} \cos \theta_{j}\right) . \eta_{s}$ is the refractive index of substrate medium. $\eta_{j}, d_{j}$, and $\theta_{j}$ are the refractive indices, thickness, and angle of incidence of the $j$ th layer, respectively.

To design an optical multilayer thin-film system should consider several practical limitations. First, according to the maximum principle [26], it is no advantage to use more than two materials that have the lowest $\eta_{l}$ and highest $\eta_{h}$ refractive indices at normal light incidence. In this case, the most general structure of multilayer system is $\left(\eta_{l} \eta_{h}\right)^{M}$ or $\left(\eta_{h} \eta_{l}\right)^{M}$. Second, the number of layers may be limited because the cost of coatings increases with the number of layers. In this paper, a layer be eliminated if its thickness is lower $0.001 \mu \mathrm{m}$. Final, the thickness cannot be negative and very thin layers are difficult controlled for some deposition processes. Therefore, the coating problem can be formulated as a constraint optimization problem. In this paper, we will use these properties to design the structures of coating systems.

\section{Family Competition Evolutionary Algorithm}

In this section, we present the detail of the family competition evolutionary algorithm (FCEA) for the optical thin-film deign. Fig. 2 shows the basic structure of the FCEA. FCEA is a multi-operator approach which integrates decreasingbased Gaussian mutation $\left(M_{d g}\right)$, self-adaptive Cauchy mutation $\left(M_{c}\right)$, and self-adaptive Gaussian mutation $\left(M_{g}\right)$. Their performance heavily depends on the same factor step size that decides the perturbation size in one step of a mutation operator.

The basic steps of the FCEA are described as follows (Fig. 2): $N$ solutions are randomly generated as the initial population. Then FCEA enters the main evolutionary loop, in which each generation consists of three nearly identical 


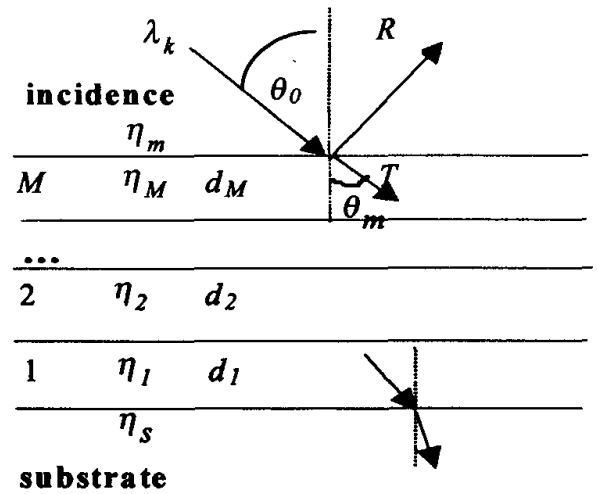

(a) The construction parameters of an optical multilayer system

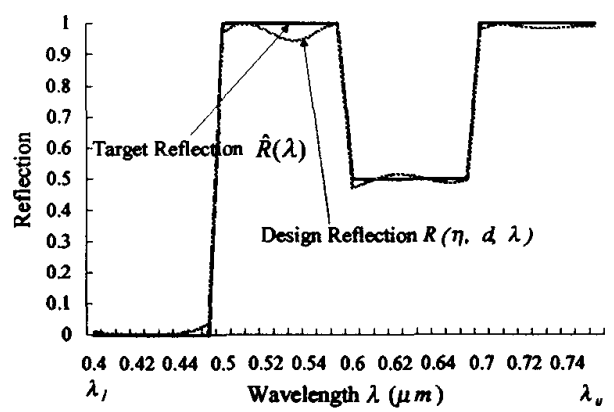

(b) Profiles of a target coating and a real construction

Figure 1: The overview of a coating system

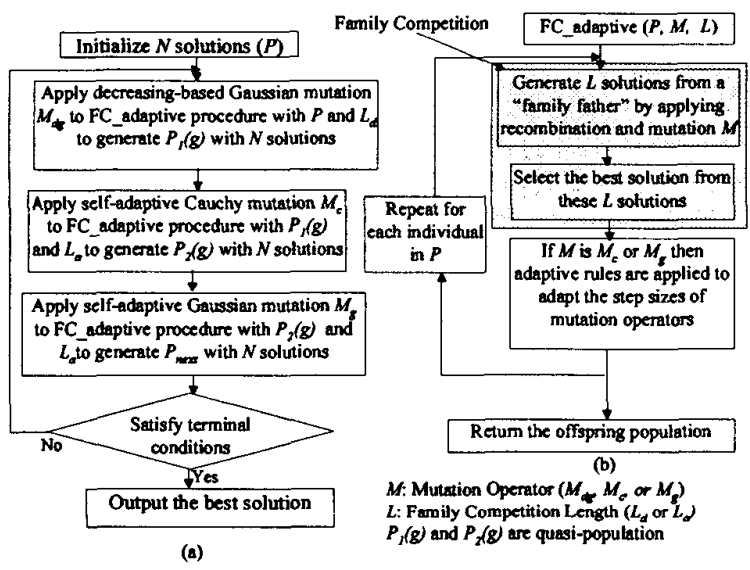

Figure 2: Overview of our algorithm: FC_adaptive procedure

(a) FCEA (b)

procedures. Each procedure is realized by doing recombinations, mutations, family competition, and selection. These three procedures mainly differ only in the mutations used: decreasing-based Gaussian mutation, self-adaptive Cauchy mutation, and self-adaptive Gaussian mutation. Hence we refer such a procedure as "FC_adaptive" which will be described later in detail. Note that the input of an FC_adaptive procedure is $N$ solutions. Then the output is a new quasipopulation with $N$ solutions which will be the input of the next FC_adaptive procedure.

The FC_adaptive procedure (2(b)) employs three parameters, namely, the parent population ( $P$ with $N$ solutions), mutation operator $(M)$, and family competition length $(L)$, to generate a new quasi-population. The main procedures of FC_adaptive are the family competition (Fig. 3) and the adaptive rules. During the family competition procedure, each in-

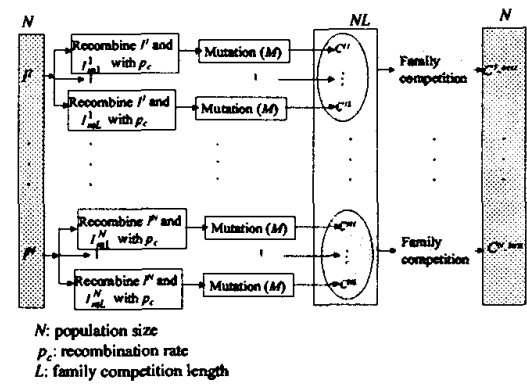

Figure 3: The main steps of the family competition

dividual $\left(I_{1}\right)$ sequentially becomes the "family father." With a probability $p_{c}$, this "family father" and another solution $\left(I_{1}^{1}\right)$ randomly chosen from the rest of the parent population are used as parents to do a recombination operation. Then the new offspring or the "family father" (if the recombination is not conducted) is operated on by a mutation to generate a offspring $\left(C^{11}\right)$. For each family father, such a procedure is repeated $L$ times. Finally $L$ solutions $\left(C^{11}, \ldots, C^{1 L}\right)$ are produced but only the one ( $C^{1 . b e s t}$ ) with the best value of merit function survives. The merit function is defined as Eq. (2). Since we create $L$ solutions from the same "family father" and perform a selection, this is a family competition strategy. We thought this was a good way to avoid the premature convergence but also to keep the spirit of local searches, because of these suspicions, and results agree [31].

Two adaptive rules are implemented to adapt step sizes in FC_adaptive procedure with self-adaptive mutations. FCEA adjusts the step sizes while mutations are applied, however, such updates may be insufficient. According to dynamic evolutionary information, adaptive rules are designed to decrease the step sizes of self-adaptive mutation or to grow the step sizes of decreasing-based mutation in order to create the rela- 
tionship of mutations after the family competition procedure.

After the FC_adaptive procedure, there are $N$ parents and $N$ children left. Based on different stages, we employ various ways of obtaining a new quasi-population with $N$ individuals. In Cauchy self-adaptive mutation stage, each pair of father and its best child the one with a better objective value survives. This procedure is called "family selection." On the other hand, "population selection" chooses the best $N$ individuals from all $N$ parents and $N$ children. With a probability $P_{p s}$, FCEA applies population selection to speed up the convergence when the decreasing-based Gaussian mutation is used. For the probability $\left(1-P_{p s}\right)$, family selection is still considered. In order to reduce the ill effects of greediness on this selection, the initial $P_{p s}$ is set to 0.05 , but it is changed to 0.5 when the mean step size of self-adaptive Cauchy mutation is larger than that of decreasing-based Gaussian mutation. Hence, through the process of selection, the FC_adaptive procedure forces each solution of the starting population to have one final offspring. Note that we create $L N$ offspring in the procedure FC_adaptive but the size of the new quasipopulation remains the same as $N$.

Regarding chromosome representation, we present each solution of a population as $(M, I, x, \sigma, v, \psi)$ where $M$ is the number of layers of a coating system. The indicator $I$ represents the structure of the refractive indices because we use only one pair of materials with $\eta_{l}$ and $\eta_{h}$. The refractive index of first layer is $\eta_{l}$ when $I$ is 0 and it is $\eta_{h}$ when $I$ is 1 . The vector $x$ is the thickness vector of a coating system to be optimized; $\sigma, v$, and $\psi$ are the step-size vectors of decreasingbased mutation, self-adaptive Gaussian mutation, and selfadaptive Cauchy mutation, respectively. In other words, each solution $x$ is associated with some parameters for step-size control. The number of element of each vector, $x, \sigma, v$, and $\psi$, is $M$. The initial value $M$ is randomly chosen from $\left[M_{l}, M_{h}\right]$ where $M_{l}$ and $M_{h}$ are the numbers of the low bound and upper bound layers, respectively. Initially, $I$ is randomly set to 1 or 0 . The initial value of each entry of $x$ is randomly chosen over a feasible region; $\sigma, v$, and $\psi$ are set to $0.04,0.01$, and 0.01 .

In the rest of this section we explain each important component of the FC_adaptive procedure: recombination operators, mutation operations, and rules for adapting step sizes $(\sigma, v$, and $\psi)$. For easy description of the operators, we use $a=\left(M^{a}, I^{a}, x^{a}, \sigma^{a}, v^{a}, \psi^{a}\right)$ to represent the "family father" and $b=\left(M^{b}, I^{b}, x^{b}, \sigma^{b}, v^{b}, \psi^{b}\right)$ as another parent (only for the recombination operator). The offspring, $c=\left(M^{c}, I^{c}, x^{c}, \sigma^{c}, v^{c}, \psi^{c}\right)$, is generated by a genetic operation. We also use the symbol $x_{j}^{d}$ to denote the thickness of $j$ th layer of a solution $d, \forall j \in\{1, \ldots, M\}$.

\subsection{Recombination Operators}

The advantages or disadvantages of a recombination for a particular objective function can hardly be accessed in advance [3]. Therefore, we implement two simple recombination operators to generate offspring: modified discrete recombina- tion and intermediate recombination [2]. Here we would like to mention again that recombination operators are activated with only a probability $p_{c}$. The optimizing solution $(x)$ and a step size $(\sigma, v$, or $\psi)$ are recombined in a recombination operator.

Modified Discrete Recombination: The original discrete recombination [2] generates a child that inherits genes from two parents with equal probability. Here the two parents of the recombination operator are the "family father" and another solution randomly selected. Our experience indicates that FCEA can be more robust if the child inherits genes from the "family father" with a higher probability [31]. Therefore, we modified the operator to be as follows:

$$
x_{j}^{c}=\left\{\begin{array}{l}
x_{j}^{a} \text { with probability } 0.8 \\
x_{j}^{b} \text { with probability } 0.2
\end{array}\right.
$$

For a "family father", applying this operator in the family competition is viewed as a local search procedure because this operator is designed to preserve the relationship between a child and its "family father".

Intermediate Recombination: We define intermediate recombination as:

$$
\begin{aligned}
x_{j}^{c} & =x_{j}^{a}+0.5\left(x_{j}^{b}-x_{j}^{a}\right), \text { and } \\
w_{j}^{c} & =w_{j}^{a}+0.5\left(w_{j}^{b}-w_{j}^{a}\right),
\end{aligned}
$$

where $w$ is $v, \sigma$, or $\psi$ based on the mutation operator applied in the family competition. For example, if self-adaptive Gaussian mutation is used in this FC_adaptive procedure. $x$ in (6) and (7) is $v$. We follow the work of the evolution strategies community [6] to employ only intermediate recombination on step-size vectors, that is, $\sigma, v$, and $\psi$.

\subsection{Mutation Operators}

Mutations are main operators of the FCEA. After the recombination, a mutation operator is applied to the "family father" or the new offspring generated by a recombination. In FCEA, the mutation is performed independently on each vector element of the selected individual by adding a random value with expectation zero:

$$
x_{j}^{\prime}=x_{j}+w D(\cdot)
$$

where $x_{j}$ is the thickness of the $j$ th layer of $x, x_{j}^{\prime}$ is the $j$ th variable of $x^{\prime}$ mutated from $x, D(\cdot)$ is a random variable, and $w$ is the step size. In this paper, $D(\cdot)$ is evaluated as $N(0,1)$ or $C(1)$ if the mutations are, respectively, Gaussian mutation or Cauchy mutation.

Self-Adaptive Gaussian Mutation: We adapted Schwefel's [24] proposal to use self-adaptive Gaussian mutation. The mutation is accomplished by first mutating the step size $v_{j}$ and then the thickness $x_{j}$ :

$$
\begin{aligned}
v_{j}^{c} & =v_{j}^{a} \exp \left[\tau^{\prime} N(0,1)+\tau N_{j}(0,1)\right], \\
x_{j}^{c} & =x_{j}^{a}+v_{j}^{c} N_{j}(0,1)
\end{aligned}
$$




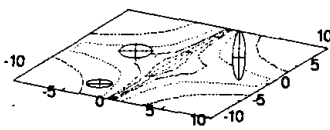

(a)

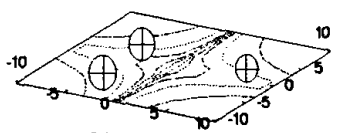

(b)

Figure 4: Difference of the search spaces between selfadaptive and decreasing-based mutations

where $N(0,1)$ is the standard normal distribution. $N_{j}(0,1)$ is a new value with distribution $N(0,1)$ that must be regenerated for each index $j$. For FCEA, we follow [6] in setting $\tau$ and $\tau^{\prime}$ as $(\sqrt{2 n})^{-1}$ and $(\sqrt{2 \sqrt{n}})^{-1}$, respectively.

Self-Adaptive Cauchy Mutation: We follow previous works [32] to define self-adaptive Cauchy mutation as follows:

$$
\begin{aligned}
\psi_{j}^{c} & =\psi_{j}^{a} \exp \left[\tau^{\prime} N(0,1)+\tau N_{j}(0,1)\right] \\
x_{j}^{c} & =x_{j}^{a}+\psi_{j}^{c} C_{j}(t)
\end{aligned}
$$

In our experiments, $t$ is 1 . Note that self-adaptive Cauchy mutation is similar to self-adaptive Gaussian mutation except that $(10)$ is replaced by (12).

Decreasing-Based Gaussian Mutations: Our decreasing-based Gaussian mutation uses the step-size vector $\sigma$ with a fixed decreasing rate $\gamma=0.97$ as follows:

$$
\begin{aligned}
& \sigma^{c}=\gamma \sigma^{a} \\
& x_{j}^{c}=x_{j}^{a}+\sigma^{c} N_{j}(0,1)
\end{aligned}
$$

Previous results [31] demonstrated that self-adaptive mutations converge faster than decreasing-based mutations but, for rugged functions, self-adaptive mutations more easily trapped into local optima than decreasing-based mutations.

For decreasing mutation, it is like we search for a better child in a hypersphere centered at the parent. However, for self-adaptive mutation, the search space becomes a hyperellipse. Fig. 4 illustrates this difference by two-dimensional contour plots. Therefore, children are searched in two different types of regions. These can be reasons why we want to use these three types of mutations.

\subsection{Adaptive Rules}

The performance of Gaussian and Cauchy mutations is largely influenced by the step sizes. FCEA adjusts the step sizes while mutations are applied (e.g. (9), (11), and (13)). However, such updates insufficiently consider the performance of the whole family. Therefore, after the family competition, some additional rules are implemented:
1. A-decrease-rule: Immediately after self-adaptive mutations, if objective values of all offspring are greater than or equal to that of the "family father," we decrease the step-size vectors $v$ (Gaussian) or $\psi$ (Cauchy) of the parent:

$$
w_{j}^{a}=0.97 w_{j}^{a},
$$

where $w^{a}$ is the step size vector of the parent. In other words, if there is no improvement after self-adaptive mutations, we may propose a more conservative be implemented. That is, smaller step size tends to make better improvement in the next iteration. This is inspired from the 1/5-success rule of $(1+\lambda)$-ES [2].

2. D-increase-rule: It is difficult, however, to decide the rate $\gamma$ of decreasing-based mutations. Unlike selfadaptive mutations which adjust step sizes automatically, its step size goes to zero as the number of iterations increases. Therefore, it is essential to employ a rule which can enlarge the step size in some situations. The step size of the decreasing-based mutation should not be too small, when compared to step sizes of selfadaptive mutations. Here, we propose to increase $\sigma$ if either of the two self-adaptive mutations generates better offspring. To be more precise, after a self-adaptive mutation, if the best child with step size $v$ is better than its "family father," the step size of the decreasing-based mutation is updated as follows:

$$
\sigma_{j}^{c}=\max \left(\sigma_{j}^{c}, \beta v_{m e a n}^{c}\right),
$$

where $v_{m e a n}^{c}$ is the mean value of the vector $v$; and $\beta$ is 0.2 in our experiments. Note that this rule is applied in stages of self-adaptive mutations but not of decreasingbased mutations.

\section{4 experimental Results}

In this section we present the numerical results for the synthesis of two different optical coatings to illustrate the proposed method. We would to mention again that all the materials were assume to be nonabsorting and nondispersive with normal incidence in this paper. Table 1 indicates the setting of FCEA parameters, such as initial step sizes, family competition lengths, and recombination probabilities. They are used for synthesis problems defined in this work. $L_{d}, \sigma$, and $p_{c D}$ are the parameters for decreasing-based mutation; $L_{a}, v$, $\psi$, and $p_{c A}$ are for self-adaptive mutations. The population size is 50 . These parameters are decided after experiments on these three optical coatings with various values.

\subsection{Infrared Antireflection Coating}

The first design problem is the synthesis of a wideband antireflection (AR) coating for germanium in the infrared. At least 60 different solutions were published for this AR coating, including non-evolutionary approaches [1], [12], [11], [14] and 
Table 1: Parameters of FCEA and notation used in this paper

\begin{tabular}{l|l}
\hline parameter name & the value and notation of parameter \\
\hline \hline recombination & $p_{c D}=0.8\left(M_{d g}\right)$ \\
probability $\left(p_{c}\right)$ & $p_{c A}=0.2\left(M_{g}\right.$ and $\left.M_{c}\right)$ \\
\hline family competition & $L_{d}=6\left(M_{d g}\right)$ \\
length & $L_{a}=6\left(M_{g}\right.$ and $\left.M_{c}\right)$ \\
\hline step sizes & $v_{i}=\psi_{i}=0.01, \sigma_{i}=4 v_{i}$ \\
\hline population size $(N)$ & 50 \\
\hline other notation & $M:$ number of layers; \\
& $L_{T}:$ total family competition length $\left(2 L_{a}+L_{d}\right) ;$ \\
& MF: value of merit function; \\
\hline
\end{tabular}

Table 2: Some solutions of FCEA for infrared antireflection coatings over the region $7.7 \leq \lambda \leq 12.3 \mu \mathrm{m}$ on a $\eta_{s}=4.0$ substrate based on the refractive index pair 2.2 and 4.2.

\begin{tabular}{lcccccccc}
\hline & S-20 & S-27 & S-33 & S-40 & S-44 & S-51 & S-61 & S-71 \\
\hline$M$ & 15 & 16 & 17 & 23 & 27 & 27 & 34 & 36 \\
$\sum \eta d(\mu \mathrm{m})$ & 20.34 & 27.04 & 33.96 & 40.17 & 44.98 & 51.19 & 61.7 & 71.15 \\
$\operatorname{MF}(\%)$ & 0.855 & 0.697 & 0.614 & 0.577 & 0.553 & 0.522 & 0.509 & 0.494 \\
\hline
\end{tabular}

evolutionary approaches [5], [22], [23]. The target design was the reflectance $R$ specified to zero at $0.1 \mu \mathrm{m}$ wavelength increments between 7.7 and $12.3 \mu \mathrm{m}$; therefore, $W$ defined in (2) is 47 . The incident medium is air $\left(\eta_{m}=1\right)$ and the substrate refractive index is $\eta_{s}=4.0$. The high- and the lowindex coating materials were $\mathrm{Ge}\left(\eta_{h}=4.2\right)$ and $\mathrm{ZnS}\left(\eta_{l}=\right.$ 2.2).

The initial number of layers is randomly chosen from 15 to 40. The initial thickness of each layer was uniformly selected from the region from 0.2 to $1.0 \mu \mathrm{m}$. FCEA totally executed 100 times and the maximum number of generations is 2000 . Table 2 shows several best solutions obtained by our FCEA on different total optical thickness.

Some of solutions from the previous research and one predicted curve by Willey [28] are shown in Fig. 5. One curve obtained by Dobrowolski [13] and believed to correspond to optimum solutions is also plotted. Fig. 5 shows that several, but by no means all, of these published solutions lie close to the believable optimum curve. Nevertheless, a number of

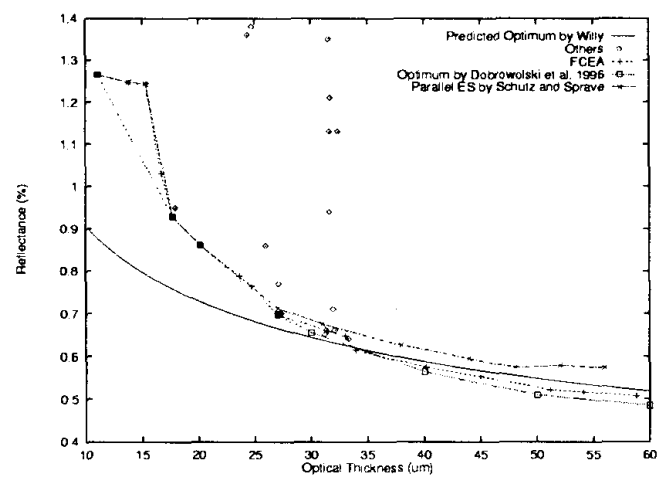

Figure 5: Comparison FCEA with Parallel ES and over 20 approaches on the antireflection coating problem. these solutions are relatively far from this curve. This implies the importance of using good starting designs or using good design techniques. Fig. 5 shows that the solutions of FCEA are very closed to the believable optimum curve. They are better than the solutions obtained by parallel evolution strategy [23] when the total thickness is larger than $35 u \mathrm{~m}$. FCEA is also very competitive with well-known approaches, such as damped least square, modified gradient, Hook and Jeeves search [1], genetic algorithm [22], and evolution strategy [5].

There are some observations according to Fig. 5.

1. Willey's predicated optimal values [28] were overestimated when the total thickness is lower than $30 \mu \mathrm{m}$. The observation was consistent with the previous findings [23], [13].

2. In [23] authors described that Willey's predicated curve [28] was the best estimate when the total thickness is beyond $30 \mu \mathrm{m}$. The solutions obtained by FCEA are better than Willey's predicated values. Therefore, we claimed that the solutions obtained in [13] are correspond to the optimum solutions for this AR coating.

3. The solution quality will be improved when the number of layer is become larger and the total thickness is thicker. We would like to mention again that the cost of coatings increases with the number of layer.

4. We observed the "Bermuda triangle", i.e. great difference between the empirical and analytical estimate [23], in the region from $11 \mu \mathrm{m}$ to $17 \mu \mathrm{m}$. The clusters of layers evident may be used to explain this observation.

\subsection{Tristimulus Filter}

The requirement of the third example is to produce a filter that matches the $\mathrm{CIE}_{\bar{x}_{\lambda}}$ curve for the standard observer in the 380-780 $\mathrm{nm}$ spectral region [25]. This filter is used in the tristimulus colorimeters. The target curve is the solid line shown in Fig. 6(a). The final designs should consist of only two coating materials $\mathrm{SiO}_{2}$ and $\mathrm{Nb}_{2} \mathrm{O}_{5}$ whose refractive indices are $\eta_{l}=1.468$ and $\eta_{h}=2.323$, respectively. Both substrate and medium are assumed to be made of glass whose index is $\eta_{s}=\eta_{m}=1.52$. The merit function was defined at 41 equispaced points on the interesting wavelength scale.

The initial number of layers is randomly chosen from 25 to 35. The initial thickness of each layer was uniformly selected from the region from 0.01 to $0.1 \mu \mathrm{m}$. The maximum number of generations is 2000 .

Fig. 6 shows a target performance and refractive-index profile of our FCEA for the $\mathrm{CIE}_{\bar{x}_{\lambda}}$ filter. The solution quality is $0.427 \%$ and the number of layers is 32 . The total thickness is $5.13 \mu \mathrm{m}$. Fig. 6(b) shows the refractive-index profile of the solution. Table 3 shows that FCEA is very comparative with needle method [25] which is a very powerful synthesis method. 


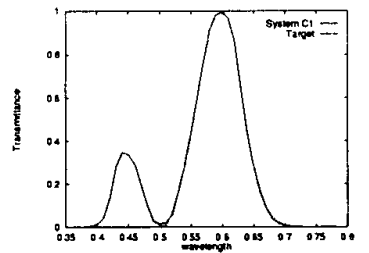

(a) target and calculated performance

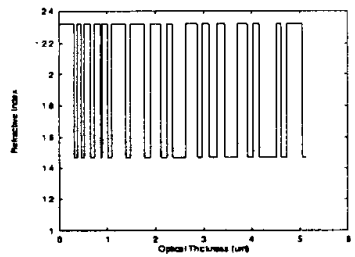

(b) refractive-index profile
Figure 6: The performance and refractive-index profile of our FCEA for the CIE ${\overline{x_{\lambda}}}_{\lambda}$ filter of the tristimulus colorimeters in the region $380-780 \mathrm{~nm}$.

Table 3: Comparison of FCEA with needle method on $\mathrm{CIE}_{\bar{x}_{\lambda}}$ filter for the tristimulus colorimeters

\begin{tabular}{c|cc|c}
\hline & \multicolumn{2}{|c|}{ FCEA } & needle method [25] \\
\hline number of layers & 32 & 34 & 31 \\
total thickness $\left(\sum \eta d\right)(\mu \mathrm{m})$ & 5.13 & 4.8 & 3.982 \\
$\operatorname{MF}(\%)$ & 0.427 & 0.588 & 0.62 \\
\hline
\end{tabular}

\section{Conclusions}

This study presents that FCEA is a stable synthesis approach for optical thin-film designs. Our experience suggests that a global optimization method should consist of both global and local search strategies. For our FCEA, the decreasingbased mutation with large initial step sizes is the global search strategy; the self-adaptive mutations with family competition procedure and replacement selection are local search strategies. Based on the family competition and adaptive rules, these mutation operators can closely cooperate with one another. Experiments of three well-known optical coating problems verify that the proposed approach is very comparative with evolutionary algorithms and traditional approaches. We believe that the flexibility and robustness of FCEA make it an effective synthesis method of optical thin-film designs.

In future, we will investigate a more flexible approach to adapt the number of layers of a coating system. We will also consider a flexible mechanism to adapt the family competition lengths for improving the performance according to the performance improvement of mutations and the morphology of the landscape.

\section{Bibliography}

[1] J. A. Aguilera, J. Aguilera, P. Baumeister, A. Bloom, D. Coursen, J. A. Dobrowolski, F. T. Goldstein, D. E. Gustafson, and R. A. Kemp. Antireflection coatings for germanium IR optics: a comparison of numerical design methods. Applied Optics, 27(14):2832-2840, 1988.

[2] T. Bäck. Evolutionary Algorithms in Theory and Practice. Oxford University Press, New York, USA, 1996.
[3] T. Bäck, U. Hammel, and H-P. Schwefel. Evolutionary computation: Comments on the history and current state. IEEE Trans. Evolutionary Computation, 1(1):3$17,1997$.

[4] T. Bäck, F. Hoffmeister, and H-P. Schwefel. A survey of evolution strategies. In Proc. Fourth Int. Conf. on Genetic Algorithms, pages 2-9, 1991.

[5] T. Bäck and M. Schütz. Evolution strategies for mixinteger optimization of optical multilayer systems. In David B. Fogel and W. Atmar, editors, Proc. 4th Annu. Conf. on Evolutionary Programming, pages 33-51. Evolutionary Programming Society, San Diego, CA. 1995.

[6] T. Bäck and H-P. Schwefel. An overview of evolution algorithms for parameter optimization. Evolutionary Computation, 1(1):1-23, 1993.

[7] B. G. Bovard. Derivation of a matrix describing a regrate dielectric thin film. Applied Optics, 27(10):19982005, 1988.

[8] J. A. Dobrowolski. Comparison of the fourier transform and flip-flop thin-film synthesis methods. Applied Opiics, 25(12):1966-1972, 1986.

[9] J. A. Dobrowolski. Merit function for more effective thin film calculations. Applied Optics, 28(14):2824 $2831,1989$.

[10] J. A. Dobrowolski. Optical properties of films and coatings. In M. Bass, editor, Handbook of Optics, chapter 42, pages 2824-2831. McGraw-Hill, New York. 1995.

[11] J. A. Dobrowolski. Numerical methods for optical thin films. Optics and Photonics News, 8(6):24-33, June 1997.

[12] J. A. Dobrowolski and R. A. Kemp. Refinement of optical multilayer systems with different optimization procedures. Applied Optics, 29(19):2876-2893, 1990.

[13] J. A. Dobrowolski, A. V. Tikhonravov, M. K. Trubetskov, B. T. Sullivan, and P. G. Verly. Optimal singleband normal-incidence antireflection coatings. Applied Optics, 35(4):644-658, 1996.

[14] J. Druessel and J. Grantham. Optimal phase modulation for gradient-index optical filters. Optics Letters, 18(19):1583-1585, 1993.

[15] T. Eisenhammer, M. Lazarov, M. Leutbecher, U. Schöffel, and R. Sizmann. Optimization of interference filters with genetic algorithms applied to silver-based heat mirrors. Applied Optics, 32(31):6310$6315,1993$. 
[16] D. B. Fogel. Evolutionary Computation: Toward a New Philosophy of Machine Intelligent. NJ:IEEE Press, Piscataway, 1995.

[17] D. E. Goldberg. Genetic Algorithms in Search, Optimization and Machine Learning. Addison-Wesley Publishing Company, Inc., Reading, MA, USA, 1989.

[18] H. Greniner. Robust optical coating design with evolutionary strategies. Applied Optics, 36(28):5477-5482, 1996.

[19] L. Li and J. A. Dobrowolski. Computation speeds of different optical thin-film synthesis methods. Applied Optics, 31(19):3790-3799, 1992.

[20] H. A. Macleod. Thin film optical filters. McGraw-Hill, New York, 1986.

[21] S. Martin, J. Rivory, and M. Schoeanauer. Simulated darwinian evolution of homogenous multilayer system: a new method for optical coating design. Optical Communication, 110:503-506, 1994.

[22] S. Martin, J. Rivory, and M. Schoeanauer. Synthesis of optical multilayer systems using genetic algorithms. Applied Optics, 34(13):2247-2254, 1995.

[23] M. Schutz and J. Sprave. Application of parallel mixedinteger evolutionary strategies with mutation rate pooling. In Proc. 5th Annu. Conf. on Evolutionary Programming, pages 345-354, 1996.

[24] H.-P. Schwefel. Numerical Optimization of Computer Models. Chichester: Wiley, 1981.

[25] B. T. Sullivan and J. A. Dobrowolski. Implementation of a numerical needle method for thin-film design. Applied Optics, 35(28):5484-5492, 1996.

[26] A. V. Tikhonravov. Some theoretical aspects of thinfilm optics and their applications. Applied Optics, 32(28):5417-5426, 1993.

[27] W. J. Wild and H. Buhay. Thin film multilayer design optimization using monte carlo approach. Optics Letters, 11(1):745-747, 1986.

[28] R. A. Willy. Predicting achievable design performance of broadband antireflective coating. Applied Optics, 32(28):5447-5451, 1993.

[29] J.-M. Yang and C.-Y. Kao. An evolutionary algorithm for synthesizing optical thin-film designs. In Parallel Problem Solving form Nature-PPSN V, (Lecture Notes in Computer Science, vol. 1498), pages 947-958, 1998.

[30] J.-M. Yang and C.-Y. Kao. Flexible ligand docking using a robust evolutionary algorithm. Journal of Computational Chemistry, to appear.
[31] J.-M. Yang and C.-Y. Kao. Integrating adaptive mutations and family competition into genetic algorithms as function optimizer. Soft Computing, 4(2), to appear.

[32] X. Yao and Y. Liu. Fast evolution strategies. In P. J. Angeline, R. G. Reynolds, J. R. McDonnell, and R. Eberhart, editors, Proc. 6th Annu. Conf. on Evolutionary Programming (Lecture Notes in Computer Science, vol. 1213), pages 151-161, 1997. 